\title{
Difference system for Selberg correlation integrals
}

\author{
Peter J. Forrester* and Masahiko Ito ${ }^{\dagger}$
}

* Department of Mathematics and Statistics, University of Melbourne, Victoria 3010, Australia;
† School of Science and Technology for future life, Tokyo Denki University, Tokyo 101-8457, Japan

\begin{abstract}
The Selberg correlation integrals are averages of the products $\prod_{s=1}^{m} \prod_{l=1}^{n}\left(x_{s}-z_{l}\right)^{\mu_{s}}$ with respect to the Selberg density. Our interest is in the case $m=1, \mu_{1}=\mu$, when this corresponds to the $\mu$-th moment of the corresponding characteristic polynomial. We give the explicit form of a $(n+1) \times(n+1)$ matrix linear difference system in the variable $\mu$ which determines the average, and we give the Gauss decomposition of the corresponding $(n+1) \times(n+1)$ matrix. For $\mu$ a positive integer the difference system can be used to efficiently compute the power series defined by this average.
\end{abstract}

\section{Introduction}

The probability density function $(\mathrm{PDF})$ on $z_{l} \in[0,1](l=1, \ldots, N)$

$$
\frac{1}{S_{n}\left(\alpha_{1}, \alpha_{2}, \tau\right)} \prod_{i=1}^{n} z_{i}^{\alpha_{1}-1}\left(1-z_{i}\right)^{\alpha_{2}-1} \prod_{1 \leq j<k \leq n}\left|z_{j}-z_{k}\right|^{2 \tau}
$$

where

$$
\begin{aligned}
S_{n}\left(\alpha_{1}, \alpha_{2}, \tau\right) & :=\int_{[0,1]^{n}} \prod_{i=1}^{n} z_{i}^{\alpha_{1}-1}\left(1-z_{i}\right)^{\alpha_{2}-1} \prod_{1 \leq j<k \leq n}\left|z_{j}-z_{k}\right|^{2 \tau} d z_{1} \cdots d z_{n} \\
& =\prod_{j=0}^{n-1} \frac{\Gamma\left(\alpha_{1}+j \tau\right) \Gamma\left(\alpha_{2}+j \tau\right) \Gamma(1+(j+1) \tau)}{\Gamma\left(\alpha_{1}+\alpha_{2}+(n+j-1) \tau\right) \Gamma(1+\tau)},
\end{aligned}
$$

is the Selberg integral, plays a fundamental role in the theories of random matrices and Calogero-Sutherland quantum many body systems (see e.g. [8]). In random matrix theory, (1) with $\left(\alpha_{1}, \alpha_{2}, \tau\right)=(a+1, b+1, \beta / 2)$ defines the Jacobi $\beta$-ensemble. For $\beta=1,2$ and 4 , and certain $a, b$, this can be realized as the eigenvalue PDF occurring in the analysis of correlation coefficients associated with Gaussian data sets, and also as the singular values of sub-matrices formed from various unitary matrices chosen according to the Haar measure. For general $\alpha_{1}, \alpha_{2}, \tau>0$ there are constructions of (1) relating to similarity reductions of unitary matrices to Hessenberg form [17], to block diagonal form [5], and to the generalized eigenvalue problem for certain tridiagonal matrices [10, 24]. 
Upon the change of variables $z_{i}=\sin ^{2} \phi_{i}, 0<\phi_{i}<\pi / 2(i=1, \ldots, n)$, the PDF (1) becomes proportional to

$$
\prod_{i=1}^{n}\left(\sin ^{2} \phi_{i}\right)^{\alpha_{1}^{\prime}}\left(\cos ^{2} \phi_{i}\right)^{\alpha_{2}^{\prime}} \prod_{1 \leq j<k \leq n}\left|\sin ^{2} \phi_{j}-\sin ^{2} \phi_{k}\right|^{2 \tau}
$$

with $\alpha_{1}^{\prime}=\alpha_{1}-1 / 2, \alpha_{2}^{\prime}=\alpha_{2}-1 / 2$. As such it is the absolute value squared of the ground state wave function for the $B C$-type Calogero-Sutherland Schrödinger operator [8, Eq.(11.55)]

$$
\begin{aligned}
-\sum_{j=1}^{n} \frac{\partial^{2}}{\partial \phi^{2}} & +\sum_{j=1}^{n}\left(\frac{\alpha_{1}^{\prime} \tau\left(\alpha_{1}^{\prime} \tau-1\right)}{\sin ^{2} \phi_{j}}+\frac{\alpha_{2}^{\prime} \tau\left(\alpha_{2}^{\prime} \tau-1\right)}{\cos ^{2} \phi_{j}}\right) \\
& +2 \tau(\tau-1) \sum_{1 \leq j<k \leq n}\left(\frac{1}{\sin ^{2}\left(\phi_{j}-\phi_{k}\right)}+\frac{1}{\sin ^{2}\left(\phi_{j}+\phi_{k}\right)}\right) .
\end{aligned}
$$

It is well known that (1) exhibits many remarkable integrability properties. One is the gamma function form of the normalization (2). Another is that the family of averages associated with (1)

$$
\int_{[0, x]^{p}} \int_{[0,1]^{n-p}} \prod_{i=1}^{n} z_{i}^{\alpha-1}\left(1-z_{i}\right)^{\beta-1}\left|x-z_{i}\right|^{\mu} \prod_{1 \leq j<k \leq n}\left|z_{j}-z_{k}\right|^{2 \tau} d z_{1} \cdots d z_{n}
$$

(we have set $\alpha_{1}=\alpha, \alpha_{2}=\beta$ ) can be characterized in terms of a certain differential-difference equation [7], equivalent to a $(n+1) \times(n+1)$ matrix Fuchsian differential equation [11, 20$]$. With $p=0$ and $\mu=2 \tau$, (3) is simply related to the one-point density implied by (1), and the differential-difference equation was used in [7] to compute the polynomial in $x$ specified by (3) in the case $\tau \in \mathbb{Z}_{+}$(the polynomial is of degree $2 \tau n$ so for practical purposes $\tau n$ cannot be too large).

In the case $\tau=1$ (3) can be calculated in terms of the solution of the Painlevé VI non-linear differential equation in $\sigma$ form [12]. It is also revealed in [12] that the $\sigma$-function associated with (3) satisfies a third order non-linear difference equation for integer shifts in the variable $\mu$, while (3) itself can be computed by a recurrence scheme based on the discrete Painlevé $\mathrm{V}$ equation. This can be understood from the viewpoint of a more general theory relating to isomonodromic deformations of linear difference systems [3].

It is the objective of this work to provide a $(n+1) \times(n+1)$ matrix linear difference system for integer shifts of the variables $\alpha, \beta$ or $\mu$ (the latter restricted to cases that $\left(x-z_{i}\right)\left|x-z_{i}\right|^{\mu}=$ $\pm\left|x-z_{i}\right|^{\mu+1}$ for some sign \pm , or alternatively to twice integer shifts) in the integrals (3) with $\tau>0$. This will be used to provide an alternative method to compute the polynomial in $x$ specified by (3) in the case $p=0, \mu-1$ even.

A precise formulation of the family of Selberg correlation integrals to be studied is given in Section 2, along with a statement of our result for the explicit form of the difference system. In Section 3 we introduce a certain family of interpolation polynomials, and we state three term relations satisfied by the corresponding generalized Selberg integrals. The three term relations are shown to imply the difference system. We give their proof in Section 4 . In Section 5 it is shown how to use the difference system to compute (3) in the polynomial case. Furthermore, we specify applied studies in random matrix theory to which our computations have relevance, and we make note too of the wider problem of characterizing correlation functions in statistical mechanical problems in terms of differential or difference equations. 


\section{Definitions and main result}

We begin with some definitions. We introduce the notation $\Phi(z), z:=\left(z_{1}, \ldots, z_{n}\right)$, to denote the generalization of the integrand (3),

$$
\Phi(z):=\prod_{i=1}^{n}\left|x_{1}-z_{i}\right|^{\alpha_{1}-1}\left|x_{2}-z_{i}\right|^{\alpha_{2}-1}\left|x_{3}-z_{i}\right|^{\alpha_{3}-1} \prod_{1 \leq j<k \leq n}\left|z_{j}-z_{k}\right|^{2 \tau}
$$

The parameters $\alpha_{1}, \alpha_{2}, \alpha_{3}$ are assumed restricted to domains for which it is possible to specify a region $\Delta \subset \mathbb{R}^{n}$ with the property $\Phi(z)$ vanishes on the boundary $\partial \Delta$ of $\Delta$. For example, if $0<x<1$ and $\operatorname{Re}\left(\alpha_{1}\right), \operatorname{Re}\left(\alpha_{2}\right), \operatorname{Re}\left(\alpha_{3}\right)>0$, we can specify

$$
\Delta=\Delta_{p}=[0, x]^{p} \times[0,1]^{n-p} \quad(p=0,1, \ldots, n) .
$$

For rational functions $\phi(z)$ bounded on $\Delta$ we define

$$
\langle\phi\rangle:=\int_{\Delta} \phi(z) \Phi(z) d z_{1} \cdots d z_{n}
$$

and we use this notation in turn to specify $T_{\alpha_{j}}$ according to

$$
T_{\alpha_{j}}\langle\phi\rangle=\left\langle\prod_{i=1}^{n}\left(z_{i}-x_{j}\right) \phi\right\rangle
$$

Note that in the cases that $\left(z_{i}-x_{j}\right)\left|z_{i}-x_{j}\right|^{\alpha_{j}-1}= \pm\left|z_{i}-x_{j}\right|^{\alpha_{j}}$ for some sign, $\pm T_{\alpha_{j}}$ corresponds to incrementing $\alpha_{j}$ by 1 , and independent of this requirement, $T_{\alpha_{j}}^{2}$ corresponds to incrementing $\alpha_{j}$ by 2 . Our goal is to identify polynomials $\left\{\varphi_{i}(z)\right\}_{i=0,1, \ldots, n+1}$ such that $\left\{T_{\alpha_{1}}\left\langle\varphi_{i}\right\rangle\right\}_{i=0,1, \ldots, n+1}$ is linearly related to $\left\{\left\langle\varphi_{i}\right\rangle\right\}_{i=0,1, \ldots, n+1}$.

For this purpose we take inspiration from the work of Aomoto [1,2]. With $\Phi^{*}(z)$ denoting $\Phi(z)$ specialized to $x_{1}=0, x_{2}=1, \alpha_{3}=1$ so that

$$
\Phi^{*}(z):=\prod_{i=1}^{n} z_{i}^{\alpha_{1}-1}\left(1-z_{i}\right)^{\alpha_{2}-1} \prod_{1 \leq j<k \leq n}\left|z_{j}-z_{k}\right|^{2 \tau}
$$

and with

$$
\langle\phi\rangle^{*}:=\int_{[0,1]^{n}} \phi(z) \Phi^{*}(z) d z_{1} \cdots d z_{n}
$$

it was proved in $[2]$ that

$$
\left\langle\prod_{l=1}^{i+1} z_{l}\right\rangle^{*}=\frac{\alpha_{1}+(n-i-1) \tau}{\alpha_{1}+\alpha_{2}+(2 n-i-2) \tau}\left\langle\prod_{l=1}^{i} z_{l}\right\rangle^{*}
$$

Since $S_{n}\left(\alpha_{1}+1, \alpha_{2}, \tau\right)=\int z_{1} \cdots z_{n} \Phi^{*}(z) d z_{1} \cdots d z_{n}$, by iterating this we immediately have the difference equation for the Selberg integral of (2),

$$
S\left(\alpha_{1}+1, \alpha_{2}, \tau\right)=S\left(\alpha_{1}, \alpha_{2}, \tau\right) \prod_{i=1}^{n} \frac{\alpha_{1}+(n-i) \tau}{\alpha_{1}+\alpha_{2}+(2 n-i-1) \tau},
$$


which in turn can be used to deduce the Gamma function evaluation given in (2). Thus we learn that in the case of the Selberg integral $T_{\alpha_{1}}\langle 1\rangle^{*}$ is linearly related to $\langle 1\rangle^{*}$, and moreover we note that to derive the linear relation use was made of the auxilary polynomials $\left\{\prod_{l=1}^{i} z_{l}\right\}$ - referred to as interpolation polynomials for the role they play in the calculation.

We will follow the same general strategy in relation to the integrals (6). Thus a family of interpolation polynomials, $\left\{\varphi_{i, j}(z)\right\}$, will be introduced so as to determine the polynomials $\left\{\varphi_{i}(z)\right\}_{i=0,1, \ldots, n+1}$ forming a polynomial basis of the difference system associated with the shift $\alpha_{1} \mapsto \alpha_{1}+1$ in the integrals (6). We know from [1,2] that the main tool in determining these polynomials is the vanishing of a certain class of averages (6).

Lemma 2.1 For $k=1,2, \ldots, n$ let

$$
\begin{aligned}
\left(\nabla_{k} \phi\right)(z) & :=\frac{\partial \phi}{\partial z_{k}}(z)+\frac{\phi(z)}{\Phi(z)} \frac{\partial \Phi}{\partial z_{k}}(z) \\
& =\frac{\partial \phi}{\partial z_{k}}(z)+\left(-\frac{\alpha_{1}-1}{x_{1}-z_{k}}-\frac{\alpha_{2}-1}{x_{2}-z_{k}}-\frac{\alpha_{3}-1}{x_{3}-z_{k}}+\sum_{\substack{1 \leq l \leq n \\
l \neq k}} \frac{2 \tau}{z_{k}-z_{l}}\right) \phi(z) .
\end{aligned}
$$

We have $\left\langle\nabla_{k} \phi\right\rangle=0$.

Proof. By definition,

$$
\left\langle\nabla_{k} \phi\right\rangle=\int_{\Delta} \Phi(z) \nabla_{k} \phi(z) d z_{1} \cdots d z_{n}=\int_{\Delta} \frac{\partial}{\partial z_{k}}(\phi(z) \Phi(z)) d z_{1} \cdots d z_{n}=0
$$

if $\phi(z) \Phi(z)$ vanishes on the boundary $\partial \Delta$ of $\Delta$, which we have assumed.

However, for purposes of presentation, rather than to start with the interpolation polynomials, it is convenient to immediately present our findings for the explicit form of the polynomials $\left\{\varphi_{i}(z)\right\}_{i=0,1, \ldots, n+1}$ and the corresponding difference system.

Theorem 2.2 Write

$$
\varphi_{i}(z):=\underbrace{\left(x_{2}-z_{1}\right) \cdots\left(x_{2}-z_{n-i}\right)}_{n-i} \times \underbrace{\left(x_{3}-z_{n-i+1}\right) \cdots\left(x_{3}-z_{n}\right)}_{i} \quad i=0,1, \ldots, n .
$$

We have

$$
T_{\alpha_{1}}\left(\left\langle\varphi_{0}\right\rangle,\left\langle\varphi_{1}\right\rangle, \ldots,\left\langle\varphi_{n}\right\rangle\right)=\left(\left\langle\varphi_{0}\right\rangle,\left\langle\varphi_{1}\right\rangle, \ldots,\left\langle\varphi_{n}\right\rangle\right) A
$$

where $A=L D U$ with

$$
L=\left(\begin{array}{cccc}
l_{00} & & \\
l_{10} & l_{11} & & \\
\cdots & \cdots & \cdots & \\
l_{n 0} & l_{n 1} & \cdots & l_{n n}
\end{array}\right), \quad D=\left(\begin{array}{llll}
d_{0} & & \\
& d_{1} & \\
& & \cdots & \\
& & & d_{n}
\end{array}\right), \quad U=\left(\begin{array}{llll}
u_{00} & u_{01} & \cdots & u_{0 n} \\
& u_{11} & \cdots & u_{1 n} \\
& & \cdots & \cdots \\
& & & u_{n n}
\end{array}\right) .
$$


All entries in $L, D, U$ not explicitly shown are zero, while for the non-zero entries we have

$$
\begin{aligned}
& l_{i j}=(-1)^{i-j}\left(\begin{array}{c}
n-j \\
n-i
\end{array}\right) \frac{\left(\alpha_{2}+j \tau ; \tau\right)_{i-j}}{\left(\alpha_{1}+\alpha_{2}+2 j \tau ; \tau\right)_{i-j}}\left(\frac{x_{2}-x_{1}}{x_{3}-x_{1}}\right)^{i-j}, \\
& d_{j}=\frac{\left(\alpha_{1} ; \tau\right)_{j}\left(\alpha_{1}+\alpha_{2}+2 j \tau ; \tau\right)_{n-j}\left(x_{2}-x_{1}\right)^{j}\left(x_{3}-x_{1}\right)^{n-j}}{\left(\alpha_{1}+\alpha_{2}+(j-1) \tau ; \tau\right)_{j}\left(\alpha_{1}+\alpha_{2}+\alpha_{3}+(n+j-1) \tau ; \tau\right)_{n-j}}, \\
& u_{i j}=(-1)^{j-i}\left(\begin{array}{l}
j \\
i
\end{array}\right) \frac{\left(\alpha_{3}+(n-j) \tau ; \tau\right)_{j-i}}{\left(\alpha_{1}+\alpha_{2}+2 i \tau ; \tau\right)_{j-i}}
\end{aligned}
$$

where $(x ; \tau)_{0}=1$ and $(x ; \tau)_{i}:=x(x+\tau)(x+2 \tau) \cdots(x+(i-1) \tau)$ for $i=1,2, \ldots$

The proof of this result will be given in Section 3 .

In Theorem 2.2 the matrix $A$ is given in terms of its Gauss $L U$ decomposition. The symmetry of (4) under the interchange $\left(x_{2}, \alpha_{2}\right) \mapsto\left(x_{3}, \alpha_{3}\right)$ allows for $A$ also to be written in terms of its $U L$ Gauss decomposition. To see this, let $\bar{L}, \bar{D}$ and $\bar{U}$ be the matrices $L, D$ and $U$ after this interchange. Since

$$
\left(\left\langle\varphi_{n}\right\rangle,\left\langle\varphi_{n-1}\right\rangle, \ldots,\left\langle\varphi_{0}\right\rangle\right)=\left(\left\langle\varphi_{0}\right\rangle,\left\langle\varphi_{1}\right\rangle, \ldots,\left\langle\varphi_{n}\right\rangle\right) J
$$

where

$$
J=\left(\begin{array}{llll} 
& & & 1 \\
& & 1 & \\
& \ldots & & \\
1 & & &
\end{array}\right)
$$

(4) can be rewritten

$$
T_{\alpha_{1}}\left(\left\langle\varphi_{0}\right\rangle,\left\langle\varphi_{1}\right\rangle, \ldots,\left\langle\varphi_{n}\right\rangle\right)=\left(\left\langle\varphi_{0}\right\rangle,\left\langle\varphi_{1}\right\rangle, \ldots,\left\langle\varphi_{n}\right\rangle\right) U^{\prime} D^{\prime} L^{\prime}
$$

where

$$
U^{\prime}=J \bar{L} J, \quad D^{\prime}=J \bar{D} J, \quad L^{\prime}=J \bar{U} J .
$$

We see that $U^{\prime}, D^{\prime}$ and $L^{\prime}$ are upper triangular, diagonal and lower triangular matrices, respectively with non-zero entries

$$
\begin{aligned}
& u_{i j}^{\prime}=(-1)^{j-i}\left(\begin{array}{l}
j \\
i
\end{array}\right) \frac{\left(\alpha_{3}+(n-j) \tau ; \tau\right)_{j-i}}{\left(\alpha_{1}+\alpha_{3}+2(n-j) \tau ; \tau\right)_{j-i}}\left(\frac{x_{3}-x_{1}}{x_{2}-x_{1}}\right)^{j-i}, \\
& d_{j}^{\prime}=\frac{\left(\alpha_{1} ; \tau\right)_{n-j}\left(\alpha_{1}+\alpha_{3}+2(n-j) \tau ; \tau\right)_{j}\left(x_{2}-x_{1}\right)^{j}\left(x_{3}-x_{1}\right)^{n-j}}{\left(\alpha_{1}+\alpha_{3}+(n-j-1) \tau ; \tau\right)_{n-j}\left(\alpha_{1}+\alpha_{2}+\alpha_{3}+(2 n-j-1) \tau ; \tau\right)_{j}} \\
& l_{i j}^{\prime}=(-1)^{i-j}\left(\begin{array}{c}
n-j \\
n-i
\end{array}\right) \frac{\left(\alpha_{2}+j \tau ; \tau\right)_{i-j}}{\left(\alpha_{1}+\alpha_{3}+2(n-i) \tau ; \tau\right)_{i-j}} .
\end{aligned}
$$




\section{Interpolation polynomials}

In this section we present some lemmas, and collaries of the lemmas, which together imply Theorem 2.2. We will defer the proof of one of these - certain key three-term relations until the next section. We begin with a lemma which enables the difference system of Theorem 2.2 to be written in a more convenient form.

Lemma 3.1 Let $U$ be as in Theorem 2.2. We have that $U^{-1}=\left(u_{i j}^{*}\right)_{0 \leq i, j \leq n}$ is the upper triangular matrix with non-zero entries

$$
u_{i j}^{*}=\left(\begin{array}{c}
j \\
i
\end{array}\right) \frac{\left(\alpha_{3}+(n-j) \tau ; \tau\right)_{j-i}}{\left(\alpha_{1}+\alpha_{2}+(j+i-1) \tau ; \tau\right)_{j-i}} .
$$

Proof. It suffices to check that for $i<j, \sum_{k=i}^{j} u_{i k} u_{k j}^{*}=0$. Now

$$
\begin{aligned}
& \sum_{k=i}^{j} u_{i k} u_{k j}^{*} \\
& \quad=\sum_{k=i}^{j}(-1)^{k-i}\left(\begin{array}{c}
k \\
i
\end{array}\right) \frac{\left(\alpha_{3}+(n-k) \tau ; \tau\right)_{k-i}\left(\begin{array}{l}
j \\
k
\end{array}\right) \frac{\left(\alpha_{3}+(n-j) \tau ; \tau\right)_{j-k}}{\left.\left(\alpha_{1}+\alpha_{2}+2 i \tau ; \tau\right)_{k-i}+\alpha_{2}+(j+k-1) \tau ; \tau\right)_{j-k}}}{\quad=\frac{\left(\alpha_{3}+(n-j) \tau ; \tau\right)_{j-i}}{\left(\alpha_{1}+\alpha_{2}+2 i \tau ; \tau\right)_{2 j-2 i-1}}\left(\begin{array}{l}
j \\
i
\end{array}\right) \sum_{k=i}^{j}(-1)^{k-i}\left(\begin{array}{c}
j-i \\
k-i
\end{array}\right)\left(\alpha_{1}+\alpha_{2}+2 i \tau+(k-i) \tau ; \tau\right)_{j-i-1}} \\
& =\frac{\left(\alpha_{3}+(n-j) \tau ; \tau\right)_{j-i}}{\left(\alpha_{1}+\alpha_{2}+2 i \tau ; \tau\right)_{2 j-2 i-1}}\left(\begin{array}{c}
j \\
i
\end{array}\right) \sum_{k=0}^{j-i}(-1)^{k}\left(\begin{array}{c}
j-i \\
k
\end{array}\right)\left(\alpha_{1}+\alpha_{2}+2 i \tau+k \tau ; \tau\right)_{j-i-1},
\end{aligned}
$$

and the last summation vanishes as an example of the summation formula for ${ }_{2} F_{1}(a, b ; c ; 1)$.

A more convenient form of the difference system can now be established.

\section{Lemma 3.2}

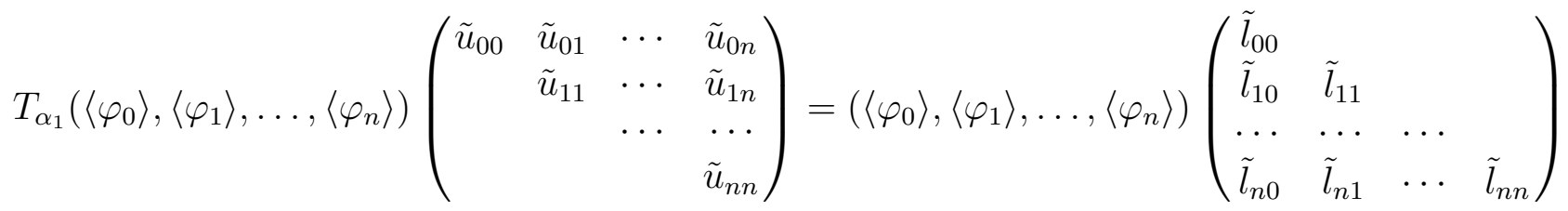

where

$$
\begin{aligned}
& \tilde{u}_{i j}=\left(\begin{array}{c}
j \\
i
\end{array}\right)\left(\alpha_{1}+\alpha_{2}+\alpha_{3}+(n+j-1) \tau ; \tau\right)_{n-j}\left(\alpha_{3}+(n-j) \tau ; \tau\right)_{j-i}\left(\alpha_{1}+\alpha_{2}+(j-1) \tau ; \tau\right)_{i}, \\
& \tilde{l}_{i j}=(-1)^{i-j}\left(\begin{array}{c}
n-j \\
n-i
\end{array}\right)\left(\alpha_{1} ; \tau\right)_{j}\left(\alpha_{2}+j \tau ; \tau\right)_{i-j}\left(\alpha_{1}+\alpha_{2}+(i+j) \tau ; \tau\right)_{n-i}\left(x_{3}-x_{1}\right)^{n-i}\left(x_{2}-x_{1}\right)^{i} .
\end{aligned}
$$

This is obtained by acting on the left of both sides of the difference system of Theorem 2.2 by $U^{-1}$, making use of the explicit form of the latter known from Lemma 3.2 on the LHS, and clearing denominators. 
Before considering the proof of this rewrite of the difference system, we make note that in a special case it implies the recurrence (10) for the Selberg integral. Thus we note from (12) that with $x_{2}=x_{3}$ we have $\varphi_{i}(z)=\prod_{i=1}^{n}\left(x_{2}-z_{i}\right)$ independent of $i$. We note too that it follows from the definitions of $\tilde{u}_{i j}$ and $\tilde{l}_{i j}$ in Lemma 3.2 that

$$
\begin{aligned}
& \sum_{i=0}^{j} \tilde{u}_{i j}=\tilde{u}_{00}=\left(\alpha_{1}+\alpha_{2}+\alpha_{3}+(n-1) \tau ; \tau\right)_{n} \\
& \left.\sum_{i=j}^{n} \tilde{l}_{i j}\right|_{x_{2}=x_{3}}=\left.\tilde{l}_{n n}\right|_{x_{2}=x_{3}}=\left(\alpha_{1} ; \tau\right)_{n}\left(x_{3}-x_{1}\right)^{n},
\end{aligned}
$$

valid for $j=0,1, \ldots, n$, where use has been made of the summation formula for ${ }_{2} F_{1}(a, b ; c ; 1)$. It follows that if we set $x_{1}=0, x_{2}=x_{3}=1$ and replace $\alpha_{2}+\alpha_{3}$ with $\alpha_{2}$ in the difference system of Lemma 3.2, then it degenerates to a single equation, which is precisely (10).

To derive the difference system of Lemma 3.2, and thus that of Theorem 2.2, we introduce the interpolation polynomials $\varphi_{i, j}(z)$ according to

$$
\varphi_{i, j}(z):=\underbrace{\left(z_{1}-x_{1}\right)\left(z_{2}-x_{1}\right) \cdots\left(z_{j}-x_{1}\right)}_{j} \varphi_{i}(z) \text { for } i, j=0,1, \ldots, n .
$$

Note that setting $j=0$ gives $\varphi_{i, 0}(x)=\varphi_{i}(z)$, while setting $j=n$ gives

$$
\varphi_{i, n}(z)=\left(z_{1}-x_{1}\right)\left(z_{2}-x_{1}\right) \cdots\left(z_{n}-x_{1}\right) \varphi_{i}(z), \quad i=0,1, \ldots, n
$$

and so

$$
T_{\alpha_{1}}\left\langle\varphi_{i}\right\rangle=\left\langle\varphi_{i, n}\right\rangle, \quad\left\langle\varphi_{i}\right\rangle=\left\langle\varphi_{i, 0}\right\rangle .
$$

Most importantly, the integrals (6) with $\phi=\varphi_{i+1, j}, \varphi_{i, j+1}, \varphi_{i+1, j+1}$, or with $\phi=\varphi_{i, j+1}, \varphi_{i, j}$, $\varphi_{i+1, j}$ satisfy certain three-term relations.

Lemma 3.3 (Three-term relations) For $i, j=0,1, \ldots, n-1$ we have

$$
\begin{aligned}
& \left(\alpha_{1}+(n-j-1) \tau\right)\left(x_{2}-x_{1}\right)\left\langle\varphi_{i+1, j}\right\rangle \\
& \quad=\left(\alpha_{3}+(n-i-1) \tau\right)\left\langle\varphi_{i, j+1}\right\rangle+\left(\alpha_{1}+\alpha_{2}+(n+i-j-1) \tau\right)\left\langle\varphi_{i+1, j+1}\right\rangle . \\
& \left(\alpha_{1}+\alpha_{2}+\alpha_{3}+(2 n-j-2) \tau\right)\left\langle\varphi_{i, j+1}\right\rangle \\
& \quad=\left(\alpha_{1}+\alpha_{2}+(n+i-j-1) \tau\right)\left(x_{3}-x_{1}\right)\left\langle\varphi_{i, j}\right\rangle-\left(\alpha_{2}+i \tau\right)\left(x_{2}-x_{1}\right)\left\langle\varphi_{i+1, j}\right\rangle .
\end{aligned}
$$

Proof. See Appendix A.

These three-term relations in fact imply the difference system of recurrences in Lemma 3.2. To see this we first use an induction on $j$ to deduce from the three-term relations two particular difference systems. 
Corollary 3.4 For $0 \leq j \leq k \leq n$ we have

$$
\begin{aligned}
& \left(\alpha_{1}+(k-j) \tau ; \tau\right)_{j}\left(x_{2}-x_{1}\right)^{j}\left\langle\varphi_{k, n-k}\right\rangle \\
& =\sum_{i=0}^{j}\left(\begin{array}{c}
j \\
i
\end{array}\right)\left(\alpha_{3}+(n-k) \tau ; \tau\right)_{j-i}\left(\alpha_{1}+\alpha_{2}+(2 k-j-1) \tau ; \tau\right)_{i}\left\langle\varphi_{i+k-j, n-k+j}\right\rangle, \\
& \left(\alpha_{1}+\alpha_{2}+\alpha_{3}+(n+j-1) \tau ; \tau\right)_{n-k}\left\langle\varphi_{j, n-j}\right\rangle \\
& =\sum_{i=k}^{n}(-1)^{i-k}\left(\begin{array}{c}
n-k \\
n-i
\end{array}\right)\left(\alpha_{1}+\alpha_{2}+(i+2 j-k) \tau ; \tau\right)_{n-i}\left(\alpha_{2}+j \tau ; \tau\right)_{i-k} \\
& \quad \times\left(x_{3}-x_{1}\right)^{n-i}\left(x_{2}-x_{1}\right)^{i-k}\left\langle\varphi_{i-k+j, k-j}\right\rangle .
\end{aligned}
$$

In particular, by setting $k=j$ in the above, for $j=0,1, \ldots, n$ we have

$$
\begin{aligned}
& \left(\alpha_{1} ; \tau\right)_{j}\left(x_{2}-x_{1}\right)^{j}\left\langle\varphi_{j, n-j}\right\rangle \\
& =\sum_{i=0}^{j}\left(\begin{array}{c}
j \\
i
\end{array}\right)\left(\alpha_{3}+(n-j) \tau ; \tau\right)_{j-i}\left(\alpha_{1}+\alpha_{2}+(j-1) \tau ; \tau\right)_{i}\left\langle\varphi_{i, n}\right\rangle, \\
& \left(\alpha_{1}+\alpha_{2}+\alpha_{3}+(n+j-1) \tau ; \tau\right)_{n-j}\left\langle\varphi_{j, n-j}\right\rangle \\
& =\sum_{i=j}^{n}(-1)^{i-j}\left(\begin{array}{c}
n-j \\
n-i
\end{array}\right)\left(\alpha_{1}+\alpha_{2}+(i+j) \tau ; \tau\right)_{n-i}\left(\alpha_{2}+j \tau ; \tau\right)_{i-j} \\
& \quad \times\left(x_{3}-x_{1}\right)^{n-i}\left(x_{2}-x_{1}\right)^{i-j}\left\langle\varphi_{i, 0}\right\rangle .
\end{aligned}
$$

Proof. See Appendix B.

The difference system of Lemma 3.2 can now be derived.

Proof of Lemma 3.2. Multiplying (21) and (22) by appropriate factors so as to make their LHS's equal, then equating RHS's gives

$$
\sum_{i=0}^{j} \tilde{u}_{i j}\left\langle\varphi_{i, n}\right\rangle=\sum_{i=j}^{n} \tilde{l}_{i j}\left\langle\varphi_{i, 0}\right\rangle, \quad j=0,1, \ldots, n
$$

where $\tilde{u}_{i j}$ and $\tilde{l}_{i j}$ are as in Lemma 3.2. Making use of (16) this reads

$$
\sum_{i=0}^{j} \tilde{u}_{i j} T_{\alpha_{1}}\left\langle\varphi_{i}\right\rangle=\sum_{i=j}^{n} \tilde{l}_{i j}\left\langle\varphi_{i}\right\rangle
$$

which is precisely the sought difference system.

\section{Implementing the recurrences}

Consider $\Phi$ specialized to the function $\Phi^{*}(z)$ of $(8)$ but with $\alpha_{1}=\alpha, \alpha_{2}=\beta$. Let this be a consequence of setting

$$
\alpha_{1}=1, \quad \alpha_{2}=\alpha, \quad \alpha_{3}=\beta, \quad x_{1}=x, \quad x_{2}=0, \quad x_{3}=1
$$


in (4). With this choice of $\Phi^{*}$ define $\langle\phi\rangle^{\#}=\langle\phi\rangle^{*} /\langle 1\rangle^{*}$, where $\langle\phi\rangle^{*}$ is specified by (9). Here our aim is to use the difference system of Theorem 2.2 to explictly compute the polynomial in $x$ specified by

$$
\left\langle\prod_{j=1}^{n}\left(x-z_{j}\right)^{\mu}\right\rangle^{\#}, \quad \mu \in \mathbb{Z}_{+}
$$

Let $\left(T_{\alpha_{1}}\left\langle\varphi_{0}\right\rangle\right)^{\#}$ refer to (7) with the substitutions (23) made afterwards, and normalized by dividing by $\langle 1\rangle^{*}$. Then according to $(7),(12)$

$$
\left(T_{\alpha_{1}}\left\langle\varphi_{0}\right\rangle\right)^{\#}=\frac{S_{n}(\alpha+1, \beta, \tau)}{S_{n}(\alpha, \beta, \tau)}\left(\left.\left\langle\prod_{j=1}^{n}\left(x-z_{j}\right)\right\rangle^{\#}\right|_{\alpha \mapsto \alpha+1}\right)
$$

Thus our task is to compute $\left(T_{\alpha_{1}}^{\mu}\left\langle\varphi_{0}\right\rangle\right)^{\#}$, as we have

$$
\left(T_{\alpha_{1}}^{\mu}\left\langle\varphi_{0}\right\rangle\right)^{\#}=(-1)^{n(\mu-1)} \frac{S_{n}(\alpha+1, \beta, \tau)}{S_{n}(\alpha, \beta, \tau)}\left(\left.\left\langle\prod_{j=1}^{n}\left(x-z_{j}\right)^{\mu}\right\rangle^{\#}\right|_{\alpha \mapsto \alpha+1}\right) .
$$

For $\mu=1$ the closed form evaluation of (24) is known from the work of Aomoto [2] as being proportional to the Jacobi polynomial $P_{N}^{\left(\gamma_{1}, \gamma_{2}\right)}(1-2 x)$ with $\gamma_{1}=\alpha / \tau-1, \gamma_{2}=\beta / \tau-1$. This in turn can be written in terms of a Gauss hypergeometric function, giving

$$
\left\langle\prod_{j=1}^{n}\left(x-z_{j}\right)\right\rangle^{\#}=\tilde{c}_{2} F_{1}(-n,(\alpha+\beta) / \tau+n-1, \alpha / \tau ; x)
$$

where

$$
\tilde{c}=\frac{(-1)^{n}(\alpha ; \tau)_{n}}{(\alpha+\beta+(n-1) \tau ; \tau)_{n}}
$$

(the factor of $\tilde{c}$ is required to make the coefficient of $x^{n}$ on the RHS unity). We can use knowledge of this to calculate $\left\langle\varphi_{k}\right\rangle^{\#}(k=0, \ldots, n)$. Once these have been determined we can use (13) with $A=: A_{\alpha_{1}}$ to recursively compute $\left(T_{\alpha_{1}}^{\mu}\left\langle\varphi_{0}\right\rangle\right) \#$ according to

$$
\left(T_{\alpha_{1}}^{\mu}\left(\left\langle\varphi_{0}\right\rangle,\left\langle\varphi_{1}\right\rangle, \ldots,\left\langle\varphi_{n}\right\rangle\right)^{\#}=\left(\left\langle\varphi_{0}\right\rangle^{\#},\left\langle\varphi_{1}\right\rangle^{\#}, \ldots,\left\langle\varphi_{n}\right\rangle^{\#}\right) A_{1} A_{2} \cdots A_{\mu}\right.
$$

Upon making use of (26) this determines $(24)$. Explicitly, with $(\vec{v})_{k}$ denoting the $k$-th component of the row vector $\vec{v}$, we have

$$
\left.\left.(-1)^{n(\mu-1)} \frac{S_{n}(\alpha+1, \beta, \tau)}{S_{n}(\alpha, \beta, \tau)}\left(\left\langle\prod_{j=1}^{n}\left(x-z_{j}\right)^{\mu}\right\rangle^{\#}\right)\right|_{\alpha \mapsto \alpha+1}\right)=\left(\left(\left\langle\varphi_{0}\right\rangle^{\#},\left\langle\varphi_{1}\right\rangle^{\#}, \ldots,\left\langle\varphi_{n}\right\rangle^{\#}\right) A_{1} A_{2} \cdots A_{\mu}\right)_{1} .
$$

Lemma 4.1 Let $\langle\phi\rangle^{\#}$ be specified as below (23). We have

$$
\left\langle\varphi_{k}\right\rangle^{\#}=(-1)^{n} \frac{(\alpha ; \tau)_{n}}{(\alpha+\beta+(n-1) \tau ; \tau)_{n}} \frac{(-\beta-(n-1) \tau ; \tau)_{k}}{(\alpha ; \tau)_{k}} .
$$


Proof. According to (7), (12) and (27)

$$
\left(T_{\alpha_{1}}\left\langle\varphi_{0}\right\rangle\right)^{\#}=\left.\frac{S_{n}(\alpha+1, \beta, \tau)}{S_{n}(\alpha, \beta, \tau)} \tilde{c}_{2} F_{1}\left(-n, 1-\beta / \tau-n, \alpha / \tau ;-\frac{x}{1-x}\right)\right|_{\alpha \mapsto \alpha+1},
$$

where use has been made of a Kummer relation for ${ }_{2} F_{1}$. Note from (10) that

$$
\frac{S_{n}(\alpha+1, \beta, \tau)}{S_{n}(\alpha, \beta, \tau)}=\frac{(\alpha ; \tau)_{n}}{(\alpha+\beta+(n-1) \tau)_{n}} .
$$

On the other hand, it follows from (13) that

$$
\left(T_{\alpha_{1}}\left\langle\varphi_{0}\right\rangle\right)^{\#}=d_{0} \sum_{k=0}^{n}\left\langle\varphi_{k}\right\rangle^{\#} l_{k 0}
$$

where, after substituting (23) in (14),

$$
d_{0} l_{k 0}=\frac{(1+\alpha ; \tau)_{n}(\alpha ; \tau)_{k}}{(1+\alpha+\beta+(n-1) \tau ; \tau)_{n}(1+\alpha ; \tau)_{k}}\left(\begin{array}{c}
n \\
n-k
\end{array}\right)\left(\frac{x}{1-x}\right)^{k} .
$$

Equating (32) and (33) we see that the factor of $(1-x)^{n}$ cancels, and we can equate coefficients of $(-x /(1-x))^{k}$ to deduce $(31)$.

It is also possible to derive (31) independent of knowledge of (27), using instead an integration formula in Jack polynomials theory, due to Warnaar [21] (see also [6]). With $\lambda=\left(\lambda_{1}, \ldots, \lambda_{n}\right)$ a partition of non-negative integers, and $P_{\lambda}^{(\alpha)}(t)=P_{\lambda}^{(\alpha)}\left(t_{1}, \ldots, t_{n}\right)$ denoting the symmetric Jack polynomial, the integration formula reads

$$
\begin{gathered}
\int_{[0, \infty)^{n}} P_{\lambda}^{(1 / \tau)}(t) \prod_{i=1}^{n} t_{i}^{x-1}\left(1+t_{i}\right)^{-x-y-2(n-1) \tau} \prod_{1 \leq j<k \leq n}\left|t_{k}-t_{j}\right|^{2 \tau} d t_{1} \cdots d t_{n} \\
=\left.P_{\lambda}^{(1 / \tau)}(v)\right|_{v_{1}=\cdots=v_{n}=-1} \frac{[x+(n-1) \tau]_{\lambda}^{(1 / \tau)}}{[-y+1]_{\lambda}^{(1 / \tau)}} S_{n}(x, y, \tau),
\end{gathered}
$$

where

$$
[u]_{\kappa}^{(\alpha)}=\prod_{j=1}^{n} \frac{\Gamma\left(u-(j-1) / \alpha+\kappa_{j}\right)}{\Gamma(u-(j-1) / \alpha)} .
$$

Our interest is in a transformed version of (35).

Corollary 4.2 Let $\langle\phi\rangle^{\#}$ be as specified below (23) and let $P_{\lambda}^{(1 / \tau)}\left(\frac{1-z}{z}\right)=P_{\lambda}^{(1 / \tau)}\left(\frac{1-z_{1}}{z_{1}}, \ldots, \frac{1-z_{n}}{z_{n}}\right)$. We have

$$
\left\langle P_{\lambda}^{(1 / \tau)}\left(\frac{1-z}{z}\right)\right\rangle^{\#}=\left.P_{\lambda}^{(1 / \tau)}(v)\right|_{v_{1}=\cdots=v_{n}=-1} \frac{[\beta+(n-1) \tau]_{\lambda}^{(1 / \tau)}}{[-\alpha+1]_{\lambda}^{(1 / \tau)}} .
$$

Proof. This follows by making the change of variables $t_{i}=\left(1-u_{i}\right) / u_{i}$ in (35), then writing $x=\beta, y=\alpha$.

For $\lambda=1^{k}$ (i.e. 1 repeated $k$ times), $k \leq n$, we have that $P_{\kappa}^{(1 / \tau)}(z)=e_{k}(z)$ where $e_{k}(z)=$ $e_{k}\left(z_{1}, \ldots, z_{n}\right)$ denotes the $k$-th elementary symmetric function. Noting from (36) that with $\kappa=1^{k}$

$$
[u]_{\kappa}^{(1 / \tau)}=(-1)^{k}(-u ; \tau)_{k}
$$

we can thus specialize (37) and so reclaim (31). 
Corollary 4.3 We have

$$
\left\langle e_{k}\left(\frac{1-z}{z}\right)\right\rangle^{\#}=\left(\begin{array}{l}
n \\
k
\end{array}\right)(-1)^{k} \frac{(-\beta-(n-1) \tau ; \tau)_{k}}{(\alpha-1 ; \tau)_{k}} .
$$

This is equivalent to (31).

Proof. It remains to explain the final assertion. This is in fact a consequence of the very definition of $\varphi_{k}$ as given in (12), which gives

$$
\begin{aligned}
\left\langle\varphi_{k}\right\rangle^{\#} & =(-1)^{n-k}\left\langle\prod_{j=1}^{n-k} z_{j} \prod_{l=n-k+1}^{n}\left(1-z_{l}\right)\right\rangle^{\#} \\
& =(-1)^{n-k}\left(\begin{array}{l}
n \\
k
\end{array}\right)^{-1} \frac{S_{n+1}(\alpha+1, \beta, \tau)}{S_{n}(\alpha, \beta, \tau)}\left(\left.\left\langle e_{k}\left(\frac{1-z}{z}\right)\right\rangle^{\#}\right|_{\alpha \mapsto \alpha+1}\right) .
\end{aligned}
$$

Substituting (38) in (39) and recalling (10) reclaims (31).

The fact that (31) has been derived independent of (27) means, from the argument of the proof of Lemma 4.1, that the difference system (13) can be used to prove (27).

With (31) substituted in (30), and the entries of $A,(14)$, specialized according to (23), we know all terms in (30) except the average - a polynomial in $x$ of degree $n \mu$ - which can therefore by computed by matrix multiplication. For example, in the case $\alpha=\beta=2, n=5$, $\tau=5$, this give for $(24)$

$$
\begin{gathered}
\frac{23}{5437500}-\frac{23 x}{65250}+\frac{3197 x^{2}}{261000}-\frac{8993 x^{3}}{56550}+\frac{2117449 x^{4}}{2035800}-\frac{793093 x^{5}}{203580} \\
+\frac{601937 x^{6}}{67860}-\frac{4384 x^{7}}{351}+\frac{7457 x^{8}}{702}-5 x^{9}+x^{10} .
\end{gathered}
$$

In general (24) in the case $\alpha=\beta$ must be unchanged (up to a possible sign) by the mapping $x=1-y$. One can check that (40) has this invariance.

We have presented the difference system (12) both for its theoretical interest, and its utility in computing the random matrix average (24) in the case $\mu \in \mathbb{Z}_{+}$. Regarding the latter, the case $\tau=1$ of (24), after the change of variables $z_{i}=\cos ^{2} \theta_{j} / 2, x=\cos ^{2} \phi / 2$, and for certain values of $a, b$, corresponds to the $\mu$-th moment of the characteristic polynomial for the classical groups $\operatorname{Sp}(2 n), O^{ \pm}(2 n)$ and $O^{ \pm}(2 n+1)$. As such it has appeared in various applied studies in random matrix theory $[9,15,16]$.

It was remarked in the Introduction that the differential-recurrence scheme of [7] can also be used to compute the random matrix average (24) in the case $\mu \in \mathbb{Z}_{+}$. This differentialrecurrence is equivalent to a $(n+1) \times(n+1)$ matrix Fuchsian differential equation $[11,20]$. The fact that there is both a differential and difference system for the Selberg correlation integrals is closely related to there being dynamical difference equations associated with solutions of the KZ equation [19]; indeed as an example of the latter theory the Selberg integral recurrence (10) was reclaimed. In recent years higher order scalar differential equations have been shown to characterize certain correlation functions in the two-dimensional Ising model [22, 23, 4] and certain generating functions in enumerative combinatorics [13, 14]. Finding characterizations of similar problems, outside the class of averages (24), in terms of higher order scalar difference equations or matrix difference equations remains an open problem. 
Another consequence of our results is in providing a fast method of computation of a certain class of generalized hypergeometric functions based on Jack polynomials $P_{\kappa}^{(\alpha)}(z)$. With $C_{\kappa}^{(\alpha)}(z):=\left(\alpha^{|\kappa|}|\kappa| ! / d_{\kappa}^{\prime}\right) P_{\kappa}^{(\alpha)}(z)$ denoting a renormalized Jack polynomial (for the definition of the quantity $d_{\kappa}^{\prime}$ see $[8$, Eq. $(12.37)]$ ), and $[u]_{\kappa}^{(\alpha)}$ defined by (36), the generalized hypergeometric functions of interest are defined by the infinite series

$$
{ }_{2} F_{1}^{(\alpha)}\left(a_{1}, a_{2} ; b_{1} ; z\right):=\sum_{\kappa} \frac{1}{|\kappa| !} \frac{\left[a_{1}\right]_{\kappa}^{(\alpha)}\left[a_{2}\right]_{\kappa}^{(\alpha)}}{\left[b_{1}\right]_{\kappa}^{(\alpha)}} C_{\kappa}^{(\alpha)}(z) .
$$

In the case $n=1$ this reduces to the usual Gauss hypergeometric function. In general, the computation of this function from the series is an inherently difficult task due to the need to sum over all partitions $\kappa$ [18]. In the special case that $a_{1}$ is a negative integer the series terminates and it is equal to a multivariable polynomial. If furthermore $z_{1}=\cdots=z_{n}=x$ (41) reduces a polynomial of degree $n\left|a_{1}\right|$ and it relates to the average (24) according to $[8$, Eq.(13.12)]

$$
\left\langle\prod_{j=1}^{n}\left(x-z_{j}\right)^{\mu}\right\rangle^{\#}=\left.x_{2}^{N \mu} F_{1}^{(\alpha)}\left(-\mu, \alpha+(n-1) \tau ; \alpha+\beta+2(n-1) \tau ; z_{1}, \ldots, z_{n}\right)\right|_{z_{1}=\cdots=z_{n}=1 / x}
$$

Thus the matrix formula (30) can be used to compute this class of ${ }_{2} F_{1}^{(\alpha)}$ using $\mathrm{O}\left(n^{3}\right)$ operations. In contrast, computation from (41) requires at least $\mathrm{O}\left(e^{\pi \sqrt{2 n / 3}}\right)$ operations, due to the sum over partitions.

\section{Acknowledgements}

PJF thanks Eric Rains for suggesting this problem. This work was supported by the Australian Research Council and JSPS Grant-in-Aid for Scientific Research (C) 21540225.

\section{A Appendix A - Proof of three-term relations}

Let $\mathfrak{S}_{n}$ be the symmetric group on $\{1,2, \ldots, n\}$, which is generated by the following reflections of the coordinates $z=\left(z_{1}, z_{2}, \ldots, z_{n}\right) \in \mathbb{C}^{n}$ :

$$
\sigma_{i, i+1}:\left(z_{1}, \ldots, z_{i}, z_{i+1}, \ldots, z_{n}\right) \mapsto\left(z_{1}, \ldots, z_{i+1}, z_{i}, \ldots, z_{n}\right), \quad i=1,2, \ldots, n-1 .
$$

For a function $f(z)$ on $\mathbb{C}^{n}$, we define action of the group $\mathfrak{S}_{n}$ on $f(z)$ by

$$
(\sigma f)(z):=f\left(\sigma^{-1}(z)\right) \quad \text { for } \quad \sigma \in \mathfrak{S}_{n} .
$$

We say that a function $f(z)$ on $\mathbb{C}^{n}$ is symmetric or skew-symmetric if $\sigma f(z)=f(z)$ or $\sigma f(z)=$ $(\operatorname{sgn} \sigma) f(z)$ for all $\sigma \in \mathfrak{S}_{n}$, respectively.

In this section, to specify the number $n$ of variables $z_{1}, z_{2}, \ldots, z_{n}$, we simply use $\varphi_{i, j}^{(n)}(z)$ instead of the polynomials $\varphi_{i, j}(z)$ defined in (15). The symbol $(n)$ on the right shoulder of $\varphi_{i, j}$ indicates the number of variables of $z=\left(z_{1}, z_{2}, \ldots, z_{n}\right)$ for $\varphi_{i, j}(z)$. 
We define the orbit of the polynomial $\varphi_{i, j}^{(n)}(z)$ by

$$
O_{i, j}^{(n)}:=\left\{\sigma \varphi_{i, j}^{(n)}(z) ; \sigma \in \mathfrak{S}_{n}\right\}
$$

and define the sum with respect to the orbit $O_{i, j}^{(n)}$ by

$$
s_{i, j}^{(n)}(z):=\sum_{\sigma \in \mathfrak{S}_{n}} \sigma \varphi_{i, j}^{(n)}(z)
$$

which is not always monic. Since, by definition, we have

$$
\left\langle\sigma \varphi_{i, j}^{(n)}\right\rangle=\left\langle\varphi_{i, j}^{(n)}\right\rangle \text { for any } \sigma \in \mathfrak{S}_{n},
$$

we obtain

$$
\left\langle s_{i, j}^{(n)}\right\rangle=n !\left\langle\varphi_{i, j}^{(n)}\right\rangle
$$

For the point $z=\left(z_{1}, z_{2}, \ldots, z_{n}\right) \in \mathbb{C}^{n}$ we set

$$
\widehat{z}_{i}=\left(z_{1}, \ldots, z_{i-1}, z_{i+1}, \ldots, z_{n}\right) \in \mathbb{C}^{n-1} \quad i=1,2, \ldots, n,
$$

so that,

$$
\begin{aligned}
& \varphi_{i, j}^{(n-1)}\left(\widehat{z}_{n}\right)=\varphi_{i, j}^{(n-1)}\left(z_{1}, z_{2}, \ldots, z_{n-1}\right) \\
& =\underbrace{\left(z_{1}-x_{1}\right) \cdots\left(z_{j}-x_{1}\right)}_{j} \times \underbrace{\left(x_{2}-z_{1}\right) \cdots\left(x_{2}-z_{n-i-1}\right)}_{n-i-1} \times \underbrace{\left(x_{3}-z_{n-i}\right) \cdots\left(x_{3}-z_{n-1}\right)}_{i}
\end{aligned}
$$

Lemma A.1 Set

$$
H(z)=H_{1}(z)+H_{2}(z)
$$

where

$$
\begin{gathered}
H_{1}(z)=-\sum_{k=1}^{n}\left[\alpha_{1}\left(x_{2}-z_{k}\right)\left(x_{3}-z_{k}\right)+\alpha_{2}\left(x_{1}-z_{k}\right)\left(x_{3}-z_{k}\right)+\alpha_{3}\left(x_{1}-z_{k}\right)\left(x_{2}-z_{k}\right)\right] s_{i, j}^{(n-1)}\left(\widehat{z}_{k}\right) \\
H_{2}(z)=\sum_{1 \leq k<l \leq n} \frac{2 \tau}{z_{k}-z_{l}}\left[\left(x_{1}-z_{k}\right)\left(x_{2}-z_{k}\right)\left(x_{3}-z_{k}\right) s_{i, j}^{(n-1)}\left(\widehat{z}_{k}\right)\right. \\
\left.-\left(x_{1}-z_{l}\right)\left(x_{2}-z_{l}\right)\left(x_{3}-z_{l}\right) s_{i, j}^{(n-1)}\left(\widehat{z}_{l}\right)\right] .
\end{gathered}
$$

Then $\langle H\rangle=0$.

Remark. Both $H_{1}(z)$ and $H_{2}(z)$ are symmetric under the action of $\mathfrak{S}_{n}$.

Proof. Put $\phi(z)=\phi_{k}(z):=\left(x_{1}-z_{k}\right)\left(x_{2}-z_{k}\right)\left(x_{3}-z_{k}\right) s_{i, j}^{(n-1)}\left(\widehat{z}_{k}\right)$ in (11) of Lemma 2.1. Since

$$
\frac{\partial \phi_{k}}{\partial z_{k}}=-\left[\left(x_{1}-z_{k}\right)\left(x_{3}-z_{k}\right)+\left(x_{1}-z_{k}\right)\left(x_{3}-z_{k}\right)+\left(x_{1}-z_{k}\right)\left(x_{2}-z_{k}\right)\right] s_{i, j}^{(n-1)}\left(\widehat{z}_{k}\right),
$$

we have $\sum_{k=1}^{n} \nabla_{k} \phi_{k}(z)=H_{1}(z)+H_{2}(z)=H(z)$. On the other hand, from Lemma 2.1, we have $\left\langle\sum_{k=1}^{n} \nabla_{k} \phi_{k}\right\rangle=\sum_{k=1}^{n}\left\langle\nabla_{k} \phi_{k}\right\rangle=0$. Therefore $\langle H\rangle=0$. 


\section{A.1 Proof of three-term relation (17)}

In this section we will prove (17) in Lemma 3.3. Throughout this section we assume that $n \leq i+j$.

First we will show $\left\langle H_{1}\right\rangle$ is expanded by $\left\langle\varphi_{i+1, j}^{(n)}\right\rangle,\left\langle\varphi_{i, j+1}^{(n)}\right\rangle$ and $\left\langle\varphi_{i+1, j+1}^{(n)}\right\rangle$. Since

$$
\begin{aligned}
& \alpha_{1}\left(x_{2}-z_{k}\right)\left(x_{3}-z_{k}\right)+\alpha_{2}\left(x_{1}-z_{k}\right)\left(x_{3}-z_{k}\right)+\alpha_{3}\left(x_{1}-z_{k}\right)\left(x_{2}-z_{k}\right) \\
& \quad=\left(\alpha_{1}+\alpha_{2}\right)\left(x_{1}-z_{k}\right)\left(x_{3}-z_{k}\right)+\alpha_{3}\left(x_{1}-z_{k}\right)\left(x_{2}-z_{k}\right)+\alpha_{1}\left(x_{2}-x_{1}\right)\left(x_{3}-z_{k}\right)
\end{aligned}
$$

and $s_{i, j}^{(n-1)}\left(\widehat{z}_{k}\right)=\sum_{\sigma \in \mathfrak{S}_{n-1}} \sigma \varphi_{i, j}^{(n-1)}\left(\widehat{z}_{k}\right)$, from $(43), H_{1}(z)$ is written as

$$
\begin{gathered}
H_{1}(z)=\sum_{k=1}^{n} \sum_{\sigma \in \mathfrak{S}_{n-1}}\left[\left(\alpha_{1}+\alpha_{2}\right)\left(z_{k}-x_{1}\right)\left(x_{3}-z_{k}\right)+\alpha_{3}\left(z_{k}-x_{1}\right)\left(x_{2}-z_{k}\right)\right. \\
\left.-\alpha_{1}\left(x_{2}-x_{1}\right)\left(x_{3}-z_{k}\right)\right] \sigma \varphi_{i, j}^{(n-1)}\left(\widehat{z}_{k}\right) .
\end{gathered}
$$

Under the assumption $n \leq i+j$, we have $n-i-1<j$. Then

$$
\begin{gathered}
\left(z_{k}-x_{1}\right)\left(x_{3}-z_{k}\right) \sigma \varphi_{i, j}^{(n-1)}\left(\widehat{z}_{k}\right) \in O_{i+1, j+1}^{(n)}, \quad\left(z_{k}-x_{1}\right)\left(x_{2}-z_{k}\right) \sigma \varphi_{i, j}^{(n-1)}\left(\widehat{z}_{k}\right) \in O_{i, j+1}^{(n)}, \\
\text { and }\left(x_{2}-x_{1}\right)\left(x_{3}-z_{k}\right) \sigma \varphi_{i, j}^{(n-1)}\left(\widehat{z}_{k}\right) \in O_{i+1, j}^{(n)} .
\end{gathered}
$$

Since $H_{1}(z)$ is symmetric, the above implies

$$
\sum_{k=1}^{n} \sum_{\sigma \in \mathfrak{S}_{n-1}}\left(z_{k}-x_{1}\right)\left(x_{3}-z_{k}\right) \sigma \varphi_{i, j}^{(n-1)}\left(\widehat{z}_{k}\right)=c s_{i+1, j+1}^{(n)}(z),
$$

where $c$ is some integer coefficient. In particular, $c=1$ is confirmed as follows. The LHS is the sum of $n \times(n-1)$ ! elements of $O_{i+1, j+1}^{(n)}$, while $s_{i+1, j+1}^{(n)}$ in the RHS is the sum of $n$ ! elements of $O_{i+1, j+1}^{(n)}$. Thus $n \times(n-1) !=n ! c$, i.e. $c=1$. In the same way we have

$$
\begin{aligned}
& \sum_{k=1}^{n} \sum_{\sigma \in \mathfrak{S}_{n-1}}\left(z_{k}-x_{1}\right)\left(x_{2}-z_{k}\right) \sigma \varphi_{i, j}^{(n-1)}\left(\widehat{z}_{k}\right)=s_{i, j+1}^{(n)}(z), \\
& \sum_{k=1}^{n} \sum_{\sigma \in \mathfrak{S}_{n-1}}\left(x_{2}-z_{k}\right)\left(x_{3}-z_{k}\right) \sigma \varphi_{i, j}^{(n-1)}\left(\widehat{z}_{k}\right)=s_{i+1, j}^{(n)}(z) .
\end{aligned}
$$

Therefore, from (45), we expand $H_{1}(z)$ as

$$
H_{1}(z)=\left(\alpha_{1}+\alpha_{2}\right) s_{i+1, j+1}^{(n)}(z)+\alpha_{3} s_{i, j+1}^{(n)}(z)-\alpha_{1}\left(x_{2}-x_{1}\right) s_{i+1, j}^{(n)}(z),
$$

so that

$$
\left\langle H_{1}\right\rangle=n !\left[\left(\alpha_{1}+\alpha_{2}\right)\left\langle\varphi_{i+1, j+1}^{(n)}\right\rangle+\alpha_{3}\left\langle\varphi_{i, j+1}^{(n)}\right\rangle-\alpha_{1}\left(x_{2}-x_{1}\right)\left\langle\varphi_{i+1, j}^{(n)}\right\rangle\right] .
$$


Next we will show $\left\langle H_{2}\right\rangle$ is expanded by $\left\langle\varphi_{i+1, j}^{(n)}\right\rangle,\left\langle\varphi_{i, j+1}^{(n)}\right\rangle$ and $\left\langle\varphi_{i+1, j+1}^{(n)}\right\rangle$. We first fix $k, l$ satisfying $1 \leq k<l \leq n$. Since, under assumption $n \leq i+j$, we have

$$
\begin{aligned}
\varphi_{i, j}^{(n-1)}\left(z_{1}, z_{2}, \ldots, z_{n-1}\right) & \\
= & \left(z_{1}-x_{1}\right) \cdots\left(z_{n-i-1}-x_{1}\right) \\
& \times \underbrace{\left(x_{2}-z_{1}\right) \cdots\left(x_{2}-z_{n-i-1}\right)}_{n-i-1} \underbrace{\left(z_{n-i}-x_{1}\right) \cdots\left(z_{j}-x_{1}\right)}_{j-(n-i-1)} \underbrace{\left(x_{3}-z_{n-i}\right) \cdots\left(x_{3}-z_{j}\right)}_{n-j-1} \underbrace{\left(x_{3}-z_{j+1}\right) \cdots\left(x_{3}-z_{n-1}\right)},
\end{aligned}
$$

if $n \leq i+j$, then for each monomial $\sigma \varphi_{i, j}^{(n-1)}\left(\widehat{z}_{k}\right) \in O_{i, j}^{(n-1)}$ one of the following three cases is chosen:

(1) $\sigma \varphi_{i, j}^{(n-1)}\left(\widehat{z}_{k}\right)$ has the factor $\left(z_{l}-x_{1}\right)\left(x_{2}-z_{l}\right)$,

(2) $\sigma \varphi_{i, j}^{(n-1)}\left(\widehat{z}_{k}\right)$ has the factor $\left(z_{l}-x_{1}\right)\left(x_{3}-z_{l}\right)$,

(3) $\sigma \varphi_{i, j}^{(n-1)}\left(\widehat{z}_{k}\right)$ has the factor $\left(x_{3}-z_{l}\right)$, but does not have the factor $\left(z_{l}-x_{1}\right)$.

Here we set $S_{i}:=\left\{\sigma \in \mathfrak{S}_{n-1} ; \sigma\right.$ satisfies the case $\left.(i)\right\}$ for $i=1,2,3$. Since the numbers of the factors $\left(z_{l}-x_{1}\right)\left(x_{2}-z_{l}\right),\left(z_{l}-x_{1}\right)\left(x_{3}-z_{l}\right)$ and $\left(x_{3}-z_{l}\right)$ appearing in $\sigma \varphi_{i, j}^{(n-1)}\left(\widehat{z}_{k}\right)$ are $n-i-1$, $j-(n-i-1)$ and $n-j-1$, respectively, we have

$$
\# S_{1}=(n-i-1)(n-2) !, \# S_{2}=(i+j-n+1)(n-2) !, \# S_{3}=(n-j-1)(n-2) !
$$

and $\# S_{1}+\# S_{2}+\# S_{3}=\# \mathfrak{S}_{n-1}=(n-1)$ !. These numbers are to be used later.

For each case we will explicitly calculate

$$
\frac{1}{z_{k}-z_{l}}\left[\left(x_{1}-z_{k}\right)\left(x_{2}-z_{k}\right)\left(x_{3}-z_{k}\right) \sigma \varphi_{i, j}^{(n-1)}\left(\widehat{z}_{k}\right)-\left(x_{1}-z_{l}\right)\left(x_{2}-z_{l}\right)\left(x_{3}-z_{l}\right) \sigma \varphi_{i, j}^{(n-1)}\left(\widehat{z}_{l}\right)\right] .
$$

The point of the calculations below is to use the identities

$$
\frac{1}{z_{k}-z_{l}}\left[\left(a-z_{k}\right)-\left(a-z_{l}\right)\right]=-1
$$

and

$$
\frac{1}{z_{k}-z_{l}}\left[\left(a-z_{k}\right)\left(b-z_{k}\right)-\left(a-z_{l}\right)\left(b-z_{l}\right)\right]=-\left[(b-a)+\left(a-z_{k}\right)+\left(a-z_{l}\right)\right] .
$$

Case 1: We assume that $\sigma \varphi_{i, j}^{(n-1)}\left(\widehat{z}_{k}\right)$ is divisible by $\left(x_{1}-z_{l}\right)\left(x_{2}-z_{l}\right)$. Then we have

$$
\frac{\sigma \varphi_{i, j}^{(n-1)}\left(\widehat{z}_{k}\right)}{\left(x_{1}-z_{l}\right)\left(x_{2}-z_{l}\right)}=\frac{\sigma \varphi_{i, j}^{(n-1)}\left(\widehat{z}_{l}\right)}{\left(x_{1}-z_{k}\right)\left(x_{2}-z_{k}\right)} .
$$

Therefore we obtain

$$
\begin{aligned}
& \frac{1}{z_{k}-z_{l}}\left[\left(x_{1}-z_{k}\right)\left(x_{2}-z_{k}\right)\left(x_{3}-z_{k}\right) \sigma \varphi_{i, j}^{(n-1)}\left(\widehat{z}_{k}\right)-\left(x_{1}-z_{l}\right)\left(x_{2}-z_{l}\right)\left(x_{3}-z_{l}\right) \sigma \varphi_{i, j}^{(n-1)}\left(\widehat{z}_{l}\right)\right] \\
& =\frac{\left(x_{1}-z_{k}\right)\left(x_{2}-z_{k}\right) \sigma \varphi_{i, j}^{(n-1)}\left(\widehat{z}_{k}\right)}{z_{k}-z_{l}}\left[\left(x_{3}-z_{k}\right)-\left(x_{3}-z_{l}\right)\right] \\
& =\left(z_{k}-x_{1}\right)\left(x_{2}-z_{k}\right) \sigma \varphi_{i, j}^{(n-1)}\left(\widehat{z}_{k}\right) \in O_{i, j+1}^{(n)} .
\end{aligned}
$$


Case 2: We assume that $\sigma \varphi_{i, j}^{(n-1)}\left(\widehat{z}_{k}\right)$ is divisible by $\left(x_{1}-z_{l}\right)\left(x_{3}-z_{l}\right)$. In the same way as Case 1 , we obtain

$$
\begin{aligned}
& \frac{1}{z_{k}-z_{l}}\left[\left(x_{1}-z_{k}\right)\left(x_{2}-z_{k}\right)\left(x_{3}-z_{k}\right) \sigma \varphi_{i, j}^{(n-1)}\left(\widehat{z}_{k}\right)-\left(x_{1}-z_{l}\right)\left(x_{2}-z_{l}\right)\left(x_{3}-z_{l}\right) \sigma \varphi_{i, j}^{(n-1)}\left(\widehat{z}_{l}\right)\right] \\
& =\left(z_{k}-x_{1}\right)\left(x_{3}-z_{k}\right) \sigma \varphi_{i, j}^{(n-1)}\left(\widehat{z}_{k}\right) \in O_{i+1, j+1}^{(n)} .
\end{aligned}
$$

Case 3: We assume that $\sigma \varphi_{i, j}^{(n-1)}\left(\widehat{z}_{k}\right)$ is divisible only by $\left(x_{3}-z_{l}\right)$. Since

$$
\frac{\sigma \varphi_{i, j}^{(n-1)}\left(\widehat{z}_{k}\right)}{\left(x_{3}-z_{l}\right)}=\frac{\sigma \varphi_{i, j}^{(n-1)}\left(\widehat{z}_{l}\right)}{\left(x_{3}-z_{k}\right)},
$$

we obtain

$$
\begin{aligned}
& \frac{1}{z_{k}-z_{l}}\left[\left(x_{1}-z_{k}\right)\left(x_{2}-z_{k}\right)\left(x_{3}-z_{k}\right) \sigma \varphi_{i, j}^{(n-1)}\left(\widehat{z}_{k}\right)-\left(x_{1}-z_{l}\right)\left(x_{2}-z_{l}\right)\left(x_{3}-z_{l}\right) \sigma \varphi_{i, j}^{(n-1)}\left(\widehat{z}_{l}\right)\right] \\
& =\frac{\left(x_{3}-z_{k}\right) \sigma \varphi_{i, j}^{(n-1)}\left(\widehat{z}_{k}\right)}{z_{k}-z_{l}}\left[\left(x_{1}-z_{k}\right)\left(x_{2}-z_{k}\right)-\left(x_{1}-z_{l}\right)\left(x_{2}-z_{l}\right)\right] \\
& =-\left(x_{3}-z_{k}\right) \sigma \varphi_{i, j}^{(n-1)}\left(\widehat{z}_{k}\right)\left[\left(x_{2}-x_{1}\right)+\left(x_{1}-z_{k}\right)+\left(x_{1}-z_{l}\right)\right] \\
& =\left(z_{k}-x_{1}\right)\left(x_{3}-z_{k}\right) \sigma \varphi_{i, j}^{(n-1)}\left(\widehat{z}_{k}\right)+\left(z_{l}-x_{1}\right)\left(x_{3}-z_{l}\right) \sigma \varphi_{i, j}^{(n-1)}\left(\widehat{z}_{l}\right) \\
& \quad-\left(x_{2}-x_{1}\right)\left(x_{3}-z_{k}\right) \sigma \varphi_{i, j}^{(n-1)}\left(\widehat{z}_{k}\right) .
\end{aligned}
$$

Note that $\left(x_{3}-z_{k}\right) \sigma \varphi_{i, j}^{(n-1)}\left(\widehat{z}_{k}\right) \in O_{i+1, j}^{(n)}$ and

$$
\left(x_{1}-z_{k}\right)\left(x_{3}-z_{k}\right) \sigma \varphi_{i, j}^{(n-1)}\left(\widehat{z}_{k}\right),\left(x_{1}-z_{l}\right)\left(x_{3}-z_{l}\right) \sigma \varphi_{i, j}^{(n-1)}\left(\widehat{z}_{l}\right) \in O_{i+1, j+1}^{(n)} .
$$

Considering the above cases, since $H_{2}(z)$ is symmetric, $H_{2}(z) / \tau$ is expanded as

$$
\frac{H_{2}(z)}{\tau}=c_{1} s_{i+1, j+1}^{(n)}(z)+c_{2} s_{i, j+1}^{(n)}(z)-\left(x_{2}-x_{1}\right) c_{3} s_{i+1, j}^{(n)}(z)
$$

where $c_{1}, c_{2}$ and $c_{3}$ are some integer coefficients. On the other hand $H_{2}(z) / \tau$ is also expanded as

$$
\begin{gathered}
\frac{H_{2}(z)}{\tau}=2 \sum_{1 \leq k<l \leq n}\left[\sum_{\sigma \in S_{1}}\left(z_{k}-x_{1}\right)\left(x_{2}-z_{k}\right) \sigma \varphi_{i, j}^{(n-1)}\left(\widehat{z}_{k}\right)+\sum_{\sigma \in S_{2}}\left(z_{k}-x_{1}\right)\left(x_{3}-z_{k}\right) \sigma \varphi_{i, j}^{(n-1)}\left(\widehat{z}_{k}\right)\right. \\
+\sum_{\sigma \in S_{3}}\left(\left(x_{1}-z_{k}\right)\left(x_{3}-z_{k}\right) \sigma \varphi_{i, j}^{(n-1)}\left(\widehat{z}_{k}\right)+\left(x_{1}-z_{l}\right)\left(x_{3}-z_{l}\right) \sigma \varphi_{i, j}^{(n-1)}\left(\widehat{z}_{l}\right)\right. \\
\left.\left.-\left(x_{2}-x_{1}\right)\left(x_{3}-z_{k}\right) \sigma \varphi_{i, j}^{(n-1)}\left(\widehat{z}_{k}\right)\right)\right] .
\end{gathered}
$$

The RHS of (52) includes the sum of $2\left(\begin{array}{c}n \\ 2\end{array}\right)\left(\# S_{2}+2 \# S_{3}\right)$ elements of $O_{i+1, j+1}^{(n)}$, while $s_{i+1, j+1}^{(n)}(z)$ in the RHS of (51) is the sum of $n$ ! elements of $O_{i+1, j+1}^{(n)}$. Thus we have $n ! c_{1}=2\left(\begin{array}{c}n \\ 2\end{array}\right)\left(\# S_{2}+2 \# S_{3}\right)$. In the same way we have $n ! c_{2}=2\left(\begin{array}{c}n \\ 2\end{array}\right) \# S_{1}$ and $n ! c_{3}=2\left(\begin{array}{c}n \\ 2\end{array}\right) \# S_{3}$. From (48), we obtain

$$
c_{1}=n-j+i-1, \quad c_{2}=n-i-1 \quad \text { and } \quad c_{3}=n-j-1 .
$$

Then (51) implies

$$
\left\langle H_{2}\right\rangle=n ! \tau\left[(n-j+i-1)\left\langle\varphi_{i+1, j+1}^{(n)}\right\rangle+(n-i-1)\left\langle\varphi_{i, j+1}^{(n)}\right\rangle-(n-j-1)\left(x_{2}-x_{1}\right)\left\langle\varphi_{i+1, j}^{(n)}\right\rangle\right] .(5
$$

From (47), (53) and Lemma A.1, i.e., $\left\langle H_{2}\right\rangle+\left\langle H_{1}\right\rangle=\langle H\rangle=0$, we therefore obtain (17). 


\section{A.2 Proof of three-term relation (18)}

In this section we will prove (18) in Lemma 3.3. Throughout this section we assume that $i+j \leq n$.

First we will show $\left\langle H_{1}\right\rangle$ is expanded by $\left\langle\varphi_{i, j}^{(n)}\right\rangle,\left\langle\varphi_{i+1, j}^{(n)}\right\rangle$ and $\left\langle\varphi_{i, j+1}^{(n)}\right\rangle$. Since

$$
\begin{aligned}
& \alpha_{1}\left(x_{2}-z_{k}\right)\left(x_{3}-z_{k}\right)+\alpha_{2}\left(x_{1}-z_{k}\right)\left(x_{3}-z_{k}\right)+\alpha_{3}\left(x_{1}-z_{k}\right)\left(x_{2}-z_{k}\right) \\
& \quad=-\left(\alpha_{1}+\alpha_{2}+\alpha_{3}\right)\left(z_{k}-x_{1}\right)\left(x_{2}-z_{k}\right)+\left(\alpha_{1}+\alpha_{2}\right)\left(x_{3}-x_{1}\right)\left(x_{2}-z_{k}\right)-\alpha_{2}\left(x_{2}-x_{1}\right)\left(x_{3}-z_{k}\right)
\end{aligned}
$$

and (43), as we gave the expression (46) of $H_{1}(z)$ in the previous section, we can expand $H_{1}(z)$ as

$$
H_{1}(z)=\left(\alpha_{1}+\alpha_{2}+\alpha_{3}\right) s_{i, j+1}^{(n)}(z)-\left(\alpha_{1}+\alpha_{2}\right)\left(x_{3}-x_{1}\right) s_{i, j}^{(n)}(z)+\alpha_{2}\left(x_{2}-x_{1}\right) s_{i+1, j}^{(n)}(z),
$$

so that

$$
\left\langle H_{1}\right\rangle=n !\left[\left(\alpha_{1}+\alpha_{2}+\alpha_{3}\right)\left\langle\varphi_{i, j+1}^{(n)}\right\rangle-\left(\alpha_{1}+\alpha_{2}\right)\left(x_{3}-x_{1}\right)\left\langle\varphi_{i, j}^{(n)}\right\rangle+\alpha_{2}\left(x_{2}-x_{1}\right)\left\langle\varphi_{i+1, j}^{(n)}\right\rangle\right] .
$$

Next we will show $\left\langle H_{2}\right\rangle$ is expanded by $\left\langle\varphi_{i, j}^{(n)}\right\rangle,\left\langle\varphi_{i+1, j}^{(n)}\right\rangle$ and $\left\langle\varphi_{i, j+1}^{(n)}\right\rangle$. We first fix $k, l$ satisfying $1 \leq k<l \leq n$. Since, under assumption $i+j \leq n$, we have

$$
\begin{aligned}
& \varphi_{i, j}^{(n-1)}\left(z_{1}, z_{2}, \ldots, z_{n-1}\right) \\
& =\left(z_{1}-x_{1}\right) \cdots\left(z_{j}-x_{1}\right) \\
& \times \underbrace{\left(x_{2}-z_{1}\right) \cdots\left(x_{2}-z_{j}\right)}_{j} \underbrace{\left(x_{2}-z_{j+1}\right) \cdots\left(x_{2}-z_{n-i}\right)}_{(n-j-1)-i} \underbrace{\left(x_{3}-z_{n-i}\right) \cdots\left(x_{3}-z_{n-1}\right)}_{i},
\end{aligned}
$$

if $i+j \leq n$, then for each monomial $\sigma \varphi_{i, j}^{(n-1)}\left(\widehat{z}_{k}\right) \in O_{i, j}^{(n-1)}$ one of the following three cases is chosen:

(1) $\sigma \varphi_{i, j}^{(n-1)}\left(\widehat{z}_{k}\right)$ has the factor $\left(z_{l}-x_{1}\right)\left(x_{2}-z_{l}\right)$,

(2) $\sigma \varphi_{i, j}^{(n-1)}\left(\widehat{z}_{k}\right)$ has the factor $\left(x_{2}-z_{l}\right)$, but does not have the factor $\left(z_{l}-x_{1}\right)$,

(3) $\sigma \varphi_{i, j}^{(n-1)}\left(\widehat{z}_{k}\right)$ has the factor $\left(x_{3}-z_{l}\right)$.

Case 1: We assume that $\sigma \varphi_{i, j}^{(n-1)}\left(\widehat{z}_{k}\right)$ is divisible by $\left(x_{1}-z_{l}\right)\left(x_{2}-z_{l}\right)$. In the same way as the previous section, from (49), we have

$$
\begin{aligned}
& \frac{1}{z_{k}-z_{l}}\left[\left(x_{1}-z_{k}\right)\left(x_{2}-z_{k}\right)\left(x_{3}-z_{k}\right) \sigma \varphi_{i, j}^{(n-1)}\left(\widehat{z}_{k}\right)-\left(x_{1}-z_{l}\right)\left(x_{2}-z_{l}\right)\left(x_{3}-z_{l}\right) \sigma \varphi_{i, j}^{(n-1)}\left(\widehat{z}_{l}\right)\right] \\
& =\left(z_{k}-x_{1}\right)\left(x_{2}-z_{k}\right) \sigma \varphi_{i, j}^{(n-1)}\left(\widehat{z}_{k}\right) \in O_{i, j+1}^{(n)} .
\end{aligned}
$$

Case 2: We assume that $\sigma \varphi_{i, j}^{(n-1)}\left(\widehat{z}_{k}\right)$ is divisible only by $\left(x_{2}-z_{l}\right)$. In the same way as the previous section, from (50), we have

$$
\begin{aligned}
& \frac{1}{z_{k}-z_{l}}\left[\left(x_{1}-z_{k}\right)\left(x_{2}-z_{k}\right)\left(x_{3}-z_{k}\right) \sigma \varphi_{i, j}^{(n-1)}\left(\widehat{z}_{k}\right)-\left(x_{1}-z_{l}\right)\left(x_{2}-z_{l}\right)\left(x_{3}-z_{l}\right) \sigma \varphi_{i, j}^{(n-1)}\left(\widehat{z}_{l}\right)\right] \\
& =\left(z_{k}-x_{1}\right)\left(x_{2}-z_{k}\right) \sigma \varphi_{i, j}^{(n-1)}\left(\widehat{z}_{k}\right)+\left(z_{l}-x_{1}\right)\left(x_{2}-z_{l}\right) \sigma \varphi_{i, j}^{(n-1)}\left(\widehat{z}_{l}\right)-\left(x_{3}-x_{1}\right)\left(x_{2}-z_{k}\right) \sigma \varphi_{i, j}^{(n-1)}\left(\widehat{z}_{k}\right) .
\end{aligned}
$$


Note that $\left(x_{2}-z_{k}\right) \sigma \varphi_{i, j}^{(n-1)}\left(\widehat{z}_{k}\right) \in O_{i, j}^{(n)}$ and

$$
\left(x_{1}-z_{k}\right)\left(x_{2}-z_{k}\right) \sigma \varphi_{i, j}^{(n-1)}\left(\widehat{z}_{k}\right),\left(x_{1}-z_{l}\right)\left(x_{2}-z_{l}\right) \sigma \varphi_{i, j}^{(n-1)}\left(\widehat{z}_{l}\right) \in O_{i, j+1}^{(n)} .
$$

$\underline{\text { Case 3: }}$ We assume that $\sigma \varphi_{i, j}^{(n-1)}\left(\widehat{z}_{k}\right)$ is divisible by $\left(x_{3}-z_{l}\right)$. Since

$$
\left(x_{1}-z_{k}\right)\left(x_{3}-z_{k}\right)=\left(x_{1}-z_{k}\right)\left(x_{2}-z_{k}\right)-\left(x_{2}-x_{1}\right)\left(x_{3}-z_{k}\right)+\left(x_{3}-x_{1}\right)\left(x_{2}-z_{k}\right),
$$

we have

$$
\begin{aligned}
\left(z_{k}-\right. & \left.x_{1}\right)\left(x_{3}-z_{k}\right) \sigma \varphi_{i, j}^{(n-1)}\left(\widehat{z}_{k}\right) \\
= & \left(z_{k}-x_{1}\right)\left(x_{2}-z_{k}\right) \sigma \varphi_{i, j}^{(n-1)}\left(\widehat{z}_{k}\right)+\left(x_{2}-x_{1}\right)\left(x_{3}-z_{k}\right) \sigma \varphi_{i, j}^{(n-1)}\left(\widehat{z}_{k}\right) \\
& \quad-\left(x_{3}-x_{1}\right)\left(x_{2}-z_{k}\right) \sigma \varphi_{i, j}^{(n-1)}\left(\widehat{z}_{k}\right) .
\end{aligned}
$$

Combining (50) and (55), we obtain

$$
\begin{aligned}
& \frac{1}{z_{k}-z_{l}}\left[\left(x_{1}-z_{k}\right)\left(x_{2}-z_{k}\right)\left(x_{3}-z_{k}\right) \sigma \varphi_{i, j}^{(n-1)}\left(\widehat{z}_{k}\right)-\left(x_{1}-z_{l}\right)\left(x_{2}-z_{l}\right)\left(x_{3}-z_{l}\right) \sigma \varphi_{i, j}^{(n-1)}\left(\widehat{z}_{l}\right)\right] \\
& =\left(z_{k}-x_{1}\right)\left(x_{3}-z_{k}\right) \sigma \varphi_{i, j}^{(n-1)}\left(\widehat{z}_{k}\right)+\left(z_{l}-x_{1}\right)\left(x_{3}-z_{l}\right) \sigma \varphi_{i, j}^{(n-1)}\left(\widehat{z}_{l}\right) \\
& \left.\quad-\left(x_{2}-x_{1}\right)\left(x_{3}-z_{k}\right) \sigma \varphi_{i, j}^{(n-1)}\left(\widehat{z}_{k}\right) \quad \text { from Eq. }(50)\right) \\
& =\left(z_{k}-x_{1}\right)\left(x_{2}-z_{k}\right) \sigma \varphi_{i, j}^{(n-1)}\left(\widehat{z}_{k}\right)-\left(x_{3}-x_{1}\right)\left(x_{2}-z_{k}\right) \sigma \varphi_{i, j}^{(n-1)}\left(\widehat{z}_{k}\right) \\
& \quad+\left(z_{l}-x_{1}\right)\left(x_{2}-z_{l}\right) \sigma \varphi_{i, j}^{(n-1)}\left(\widehat{z}_{l}\right)+\left(x_{2}-x_{1}\right)\left(x_{3}-z_{l}\right) \sigma \varphi_{i, j}^{(n-1)}\left(\widehat{z}_{l}\right) \\
& \left.\quad-\left(x_{3}-x_{1}\right)\left(x_{2}-z_{l}\right) \sigma \varphi_{i, j}^{(n-1)}\left(\widehat{z}_{l}\right) \quad \text { (from Eq. }(55)\right) .
\end{aligned}
$$

Here

$$
\begin{gathered}
\left(z_{k}-x_{1}\right)\left(x_{2}-z_{k}\right) \sigma \varphi_{i, j}^{(n-1)}\left(\widehat{z}_{k}\right),\left(z_{k}-x_{1}\right)\left(x_{2}-z_{l}\right) \sigma \varphi_{i, j}^{(n-1)}\left(\widehat{z}_{l}\right) \in O_{i, j+1}^{(n)}, \\
\left(x_{2}-z_{k}\right) \sigma \varphi_{i, j}^{(n-1)}\left(\widehat{z}_{k}\right),\left(x_{2}-z_{l}\right) \sigma \varphi_{i, j}^{(n-1)}\left(\widehat{z}_{l}\right) \in O_{i, j}^{(n)}, \\
\left(x_{3}-z_{l}\right) \sigma \varphi_{i, j}^{(n-1)}\left(\widehat{z}_{l}\right) \in O_{i+1, j}^{(n)} .
\end{gathered}
$$

Considering the above cases, since the numbers of the factors $\left(z_{l}-x_{1}\right)\left(x_{2}-z_{l}\right),\left(x_{2}-z_{l}\right)$ and $\left(x_{3}-z_{l}\right)$ appearing in $\sigma \varphi_{i, j}^{(n-1)}\left(\widehat{z}_{k}\right)$ are $j,(n-j-1)-i$ and $i$, respectively, as we saw in the previous section, the function $H_{2}(z)$ is expanded in total as

$$
\begin{aligned}
H_{2}(z)= & \frac{2 \tau\left(\begin{array}{c}
n \\
2
\end{array}\right)(n-2) !}{n !}\left[j s_{i, j+1}^{(n)}(z)+(n-i-j-1)\left(2 s_{i, j+1}^{(n)}(z)-\left(x_{3}-x_{1}\right) s_{i, j}^{(n)}(z)\right)\right. \\
& \left.+i\left(2 s_{i, j+1}^{(n)}(z)-2\left(x_{3}-x_{1}\right) s_{i, j}^{(n)}(z)+\left(x_{2}-x_{1}\right) s_{i+1, j}^{(n)}(z)\right)\right] \\
= & \tau\left[(2 n-j-2) s_{i, j+1}^{(n)}(z)-(n+i-j-1)\left(x_{3}-x_{1}\right) s_{i, j}^{(n)}(z)+i\left(x_{2}-x_{1}\right) s_{i+1, j}^{(n)}(z)\right],
\end{aligned}
$$

so that

$$
\left\langle H_{2}\right\rangle=n ! \tau\left[(2 n-j-2)\left\langle\varphi_{i, j+1}^{(n)}\right\rangle-(n+i-j-1)\left(x_{3}-x_{1}\right)\left\langle\varphi_{i, j}^{(n)}\right\rangle+i\left(x_{2}-x_{1}\right)\left\langle\varphi_{i+1, j}^{(n)}\right\rangle\right] .
$$

From (54), (56) and Lemma A.1, i.e., $\left\langle H_{2}\right\rangle+\left\langle H_{1}\right\rangle=\langle H\rangle=0$, we therefore obtain (18). 


\section{B Appendix B - Proof of Corollary 3.4}

We detail the case of Eq. (19) only; the proof of (20) proceeds similarly.

Induction on $j$ : If $j=1$, then Eq. (19) reads

$\left(\alpha_{1}+(k-1) \tau\right)\left(x_{2}-x_{1}\right)\left\langle\varphi_{k, n-k}\right\rangle=\left(\alpha_{3}+(n-k) \tau\right)\left\langle\varphi_{k-1, n-k+1}\right\rangle+\left(\alpha_{1}+\alpha_{2}+(2 k-2) \tau\right)\left\langle\varphi_{k, n-k+1}\right\rangle$,

which is confirmed from (17) by setting $i=k-1$ and $j=n-k$. Next we assume the following as an inductive hypothesis

$$
\begin{aligned}
& \left(\alpha_{1}+(k-j+1) \tau ; \tau\right)_{j-1}\left(x_{2}-x_{1}\right)^{j-1}\left\langle\varphi_{k, n-k}\right\rangle \\
& \quad=\sum_{i=0}^{j-1}\left(\begin{array}{c}
j-1 \\
i
\end{array}\right)\left(\alpha_{3}+(n-k) \tau ; \tau\right)_{j-i-1}\left(\alpha_{1}+\alpha_{2}+(2 k-j) \tau ; \tau\right)_{i}\left\langle\varphi_{i+k-j+1, n-k+j-1}\right\rangle .
\end{aligned}
$$

On the other hand, from (17) by setting $i \rightarrow i+k-j$ and $j \rightarrow n-k+j-1$ we have

$$
\begin{aligned}
& \left(\alpha_{1}+(k-j) \tau\right)\left(x_{2}-x_{1}\right)\left\langle\varphi_{i+k-j+1, n-k+j-1}\right\rangle \\
& \quad=\left(\alpha_{3}+(n-i-k+j-1) \tau\right)\left\langle\varphi_{i+k-j, n-k+j}\right\rangle+\left(\alpha_{1}+\alpha_{2}+(i+2 k-2 j) \tau\right)\left\langle\varphi_{i+k-j+1, n-k+j}\right\rangle .
\end{aligned}
$$

Then the LHS of (19) is written as

$$
\begin{aligned}
& \left(\alpha_{1}+(k-j) \tau ; \tau\right)_{j}\left(x_{2}-x_{1}\right)^{j}\left\langle\varphi_{k, n-k}\right\rangle \\
& =\left(\alpha_{1}+(k-j) \tau\right)\left(x_{2}-x_{1}\right) \\
& \times \sum_{i=0}^{j-1}\left(\begin{array}{c}
j-1 \\
i
\end{array}\right)\left(\alpha_{3}+(n-k) \tau ; \tau\right)_{j-i-1}\left(\alpha_{1}+\alpha_{2}+(2 k-j) \tau ; \tau\right)_{i}\left\langle\varphi_{i+k-j+1, n-k+j-1}\right\rangle \\
& \text { (from (57)) } \\
& =\sum_{i=0}^{j-1}\left(\begin{array}{c}
j-1 \\
i
\end{array}\right)\left(\alpha_{3}+(n-k) \tau ; \tau\right)_{j-i-1}\left(\alpha_{1}+\alpha_{2}+(2 k-j) \tau ; \tau\right)_{i} \\
& \times\left[\left(\alpha_{3}+(n-i-k+j-1) \tau\right)\left\langle\varphi_{i+k-j, n-k+j}\right\rangle\right. \\
& \left.+\left(\alpha_{1}+\alpha_{2}+(i+2 k-2 j) \tau\right)\left\langle\varphi_{i+k-j+1, n-k+j}\right\rangle\right] \\
& =\sum_{i=0}^{j-1}\left(\begin{array}{c}
j-1 \\
i
\end{array}\right)\left(\alpha_{3}+(n-k) \tau ; \tau\right)_{j-i-1}\left(\alpha_{1}+\alpha_{2}+(2 k-j) \tau ; \tau\right)_{i} \\
& \times\left(\alpha_{3}+(n-i-k+j-1) \tau\right)\left\langle\varphi_{i+k-j, n-k+j}\right\rangle \\
& +\sum_{i=1}^{j}\left(\begin{array}{c}
j-1 \\
i-1
\end{array}\right)\left(\alpha_{3}+(n-k) \tau ; \tau\right)_{j-i}\left(\alpha_{1}+\alpha_{2}+(2 k-j) \tau ; \tau\right)_{i-1} \\
& \times\left(\alpha_{1}+\alpha_{2}+(i+2 k-2 j-1) \tau\right)\left\langle\varphi_{i+k-j, n-k+j}\right\rangle \\
& =\sum_{i=0}^{j}\left(\alpha_{3}+(n-k) \tau ; \tau\right)_{j-i}\left(\alpha_{1}+\alpha_{2}+(2 k-j) \tau ; \tau\right)_{i-1} \\
& \times\left[\left(\begin{array}{c}
j-1 \\
i
\end{array}\right)\left(\alpha_{1}+\alpha_{2}+(2 k-j+i-1) \tau\right)+\left(\begin{array}{c}
j-1 \\
i-1
\end{array}\right)\left(\alpha_{1}+\alpha_{2}+(i+2 k-2 j-1) \tau\right)\right] \\
& \times\left\langle\varphi_{i+k-j, n-k+j}\right\rangle \text {. }
\end{aligned}
$$


Here

$$
\begin{aligned}
& \left(\begin{array}{c}
j-1 \\
i
\end{array}\right)\left(\alpha_{1}+\alpha_{2}+(2 k-j+i-1) \tau\right)+\left(\begin{array}{c}
j-1 \\
i-1
\end{array}\right)\left(\alpha_{1}+\alpha_{2}+(i+2 k-2 j-1) \tau\right) \\
& =\left[\left(\begin{array}{c}
j-1 \\
i
\end{array}\right)+\left(\begin{array}{c}
j-1 \\
i-1
\end{array}\right)\right]\left(\alpha_{1}+\alpha_{2}+(2 k-j+i-1) \tau\right)-j\left(\begin{array}{c}
j-1 \\
i-1
\end{array}\right) \tau \\
& =\left(\begin{array}{c}
j \\
i
\end{array}\right)\left(\alpha_{1}+\alpha_{2}+(2 k-j+i-1) \tau\right)-i\left(\begin{array}{l}
j \\
i
\end{array}\right) \tau=\left(\begin{array}{l}
j \\
i
\end{array}\right)\left(\alpha_{1}+\alpha_{2}+(2 k-j-1) \tau\right) .
\end{aligned}
$$

From (59) and (60) we obtain Eq. (19).

\section{References}

[1] K. Aomoto, On vanishing of cohomology attached to certain many valued meromorphic functions, J. Math. Soc. Japan 27 (1975), 127-133.

[2] _ Jacobi polynomials associated with Selberg's integral, SIAM J. Math. Analysis 18 (1987), 545-549.

[3] A. Borodin, Isomonodromic transformations of linear systems of difference equations, Ann. Math. 160 (2004), 1141-1182.

[4] A. Bostan, S. Boukraa, A.J. Guttmann, S. Hassani, I. Jensen, J.M. Maillard and N. Zenine, High order Fuchsian equations for the square lattice Ising model: $\tilde{\chi}^{(5)}$, J. Phys. A 42 (2009), 275209 (32pp)

[5] A. Edelman and B.D. Sutton, The beta-Jacobi matrix model, the CS decomposition, and generalized singular value problems, Found. Comput. Math. 8 (2008), 259-285.

[6] Z.M. Feng and J.P. Song, Integrals over the circular ensembles relating to classical domains, J. Phys. A 42 (2009), 325204 (23pp).

[7] P.J. Forrester, Recurrence equations for the computation of correlations in the $1 / r^{2}$ quantum many body system, J. Stat. Phys. 72 (1993), 39-50.

[8] L Log-gases and Random Matrices, Princeton University Press, Princeton 2010.

[9] P.J. Forrester and J.P. Keating, Singularity dominated strong fluctuations for some random matrix averages, Commun. Math. Phys. 250 (2004), 119-131.

[10] P.J. Forrester and E.M. Rains, Interpretations of some parameter dependent generalizations of classical matrix ensembles, Prob. Theory Related Fields 131 (2005), 1-61.

[11] P.J. Forrester and S.O. Warnaar, The importance of the Selberg integral, Bull. Am. Math. Soc. 45 (2008), 489-534.

[12] P.J. Forrester and N.S. Witte, Application of the $\tau$-function theory of Painlevé equations to random matrices: PVI, the JUE, CyUE, cJUE and scaled limits, Nagoya Math. J. 174 (2004), 29-114. 
[13] A.J. Guttmann and I Jensen, The perimeter generating function of punctured staircase polygons, J. Phys. A 39 (2006), 3871-3882.

[14] I. Jensen and A. Rechnitzer, The exact perimeter generating function for a model of punctured staircase polygons, J. Phys. A 41 (2008), 215002 (12pp).

[15] J.P. Keating and F. Mezzadri, Random matrix theory and entanglement in quantum spin chains, Commun. Math. Phys. 252 (2004), 543-579.

[16] J.P. Keating and B.E. Odgers, Symmetry transitions in random matrix theory and Lfunctions, Commun. Math. Phys. 281 (2008), 499-528.

[17] R. Killip and I. Nenciu, Matrix models for circular ensembles, Int. Math. Res. Not. 50 (2004), 2665-2701.

[18] P. Koev and A. Edelman, The efficient evaluation of the hypergeometric function of a matrix argument, Math. Comp. 75 (2006), 833-846.

[19] Y. Markov and A. Varchenko, Hypergeometric solutions of trigonometric KZ equations satisfy dynamical difference equations, Adv. Math. 166, 100-147.

[20] K. Mimachi, The connection problem associated with a Selberg type integral and the qracah polynomials, arXiv:0710.2167, 2007.

[21] S.O. Warnaar, q-Selberg integrals and Macdonald polynomials, Ramanujan J. 10 (2005), 237-268.

[22] N. Zenine, S. Boukraa, S. Hassani and J.M. Maillard, The Fuchsian differential equation of the square lattice Ising model $\chi^{(3)}$ susceptibility, J. Phys. A 37 (2004), 9651-9668.

[23] Ising model susceptibility: the Fuchsian differential equation for $\chi^{(4)}$ and its factorization properties, J. Phys. A 38 (2005), 4149-4173

[24] A. Zhedanov, Biorthogonal rational functions and the generalized eigenvalue problem, J. Approx. Th. 101 (1999), 303-329. 